\title{
Dynamic light scattering studies of the effects of salts on the diffusivity of cationic and anionic cavitands
}

\author{
Anthony Wishard and Bruce C. Gibb ${ }^{*}$
}

\author{
Full Research Paper \\ Address: \\ Department of Chemistry, Tulane University, New Orleans, LA 70118, \\ USA \\ Email: \\ Bruce C. Gibb* - bgibb@tulane.edu \\ * Corresponding author \\ Keywords: \\ cavitand; dynamic light scattering; Hofmeister effect; ion-ion \\ interactions; water
}

\author{
Beilstein J. Org. Chem. 2018, 14, 2212-2219. \\ doi:10.3762/bjoc. 14.195 \\ Received: 04 May 2018 \\ Accepted: 15 August 2018 \\ Published: 23 August 2018 \\ This article is part of the thematic issue "Macrocyclic and supramolecular \\ chemistry". \\ Guest Editor: M.-X. Wang \\ (C) 2018 Wishard and Gibb; licensee Beilstein-Institut. \\ License and terms: see end of document.
}

\begin{abstract}
Although alkali halide salts play key roles in all living systems, the physical models used to describe the properties of aqueous solutions of salts do not take into account specific ion-ion interactions. To identify specific ion-ion interactions possibly contributing to the aggregation of proteins, we have used dynamic light scattering (DLS) to probe the aggregation of charged cavitands. DLS measurements of negatively charged $\mathbf{1}$ in the presence of a range of alkali metal halides reveal no significant aggregation of host $\mathbf{1}$ as a function of the nature of the cation of the added salt. Only at high concentrations could trace amounts of aggregation be detected by ${ }^{1} \mathrm{H}$ NMR spectroscopy. Contrarily, 1 was readily aggregated and precipitated by $\mathrm{ZnCl}_{2}$. In contrast, although fluoride and chloride did not induce aggregation of positively charged host $\mathbf{2}$, this cavitand exhibited marked aggregation as a function of bromide and iodide concentration. Specifically, bromide induced small but significant amounts of dimerization, whilst iodide induced extreme aggregation. Moreover, in these cases aggregation of host $\mathbf{2}$ also exhibited a cationic dependence, with an observed trend $\mathrm{Na}^{+}>\mathrm{Li}^{+}>\mathrm{K}^{+} \approx \mathrm{Cs}^{+}$. In combination, these results reveal new details of specific ion pairings in aqueous solution and how this can influence the properties of dissolved organics.
\end{abstract}

\section{Introduction}

Although all life on planet Earth depends on aqueous solutions, our understanding of aqueous supramolecular chemistry is limited. As a result, as Smith has eloquently pointed out [1], the effects of buffers and salts on dissolved organics can be quite bewildering. Why is this? The proverbial elephant in the room is that classical theories of electrolytes rest on the assumptions that all ions are point charges that only form non-specific interactions. The ramifications of this are innumerable. For example, pH measurements are based on extended Debye-Hückel theory [2] and Poisson-Boltzmann distribution [3] to describe ionic 
profiles near the glass-electrode surface. These classical models may be good approximations for ions such as $\mathrm{Li}^{+}$and $\mathrm{F}^{-}$, but they are poor models for ions that don't behave as hard point charges [4]. Correspondingly, IUPAC advises researchers to avoid $\mathrm{pH}$ measurements above $0.1 \mathrm{M}$ to minimize errors [5] Related problems lie with Derjaguin, Landau, Verwey and Overbeek (DLVO) theory as a model of the aggregation of aqueous dispersions. DLVO often quantitatively succeeds, but it fails to predict ion specific effects $[4,6,7]$. Similarly, it is becoming increasingly evident that the Hofmeister and reverse Hofmeister effects $[8,9]$ - most commonly discussed in terms of how salts affect biomacromolecules - can only be fully understood in terms of specific ion-ion, ion-water, and/or ion-macromolecule interactions $[4,10,11]$.

Although many attempts have been made to amend these and other classical models [4], success has been limited because of our lack of understanding of the specific supramolecular properties of individual ions. There is therefore an opportunity for supramolecular chemists (who by their very training demand specificity of interactions) to help build a full understanding of ion-specific interactions in water and help usher the troubling elephant out of the room.

Recently we demonstrated how host molecules can engender the Hofmeister [12,13] and the reverse Hofmeister effects [14]. In regards to the former, we have shown how poorly solvated anions such as $\mathrm{SCN}^{-}$have an affinity for non-polar surfaces. Because of this, they can compete with the interactions between two non-polar surfaces in a host-guest complexation event and can induce an apparent weakening of the hydrophobic effect akin to how these anions can partially unfold proteins. Alternatively, poorly solvated anions can also associate closely with cationic groups, induce charge neutralization, and engender aggregation and/or precipitation. In other words, they can also cause an apparent increase in the hydrophobic effect. This is the reverse Hofmeister effect, and in complex biomacromolecules we surmise that both effects are in operation, and that in very general terms it is the balance between these that dictates the properties of a particular macromolecule under specific conditions. Cations can also induce Hofmeister effects, but these are usually much weaker, and we believe there are two reasons for this. First, simple metal cations are generally more strongly solvated than comparable anions that can induce Hofmeister effects. Second, the anions that predominate in biomacromolecules are carboxylates, phosphates and sulfates, and the strong solvation of these means that it is hard for a cation to form an ion pair and induce Hofmeister effects.

To explore these ideas further we report here the responses of two deep-cavity cavitands, octacarboxylate $\mathbf{1}$ (counter ion $\mathrm{Na}^{+}$) $[15,16]$ and positand 2 (counter ion $\mathrm{Cl}^{-}$) [14] (Figure 1), to different salts using dynamic light scattering (DLS) [17-20]. Respectively functionalized with carboxylates and trimethylammonium groups, these hosts are expected to possess unique ionpairing properties and hence have very different reverseHofmeister responses to added salts. More specifically, both octacarboxylate $\mathbf{1}$ and positand $\mathbf{2}$ have a non-polar cavity that can function as an anion (but not to our knowledge a cation) binding site. Anion binding to the cavity of positively charged 2 is stronger than to negatively charged $\mathbf{1}[13,14]$, but nevertheless anion binding to 1 can be as strong as $4.60 \mathrm{kcal} \mathrm{mol}^{-1}$. Host 2 has a second anion binding site in the form of the crown of trimethylammoniums "under" the primary bowl [14], and correspondingly the four chelating carboxylates of the crown of $\mathbf{1}$ may be a reasonable cation binding site. Furthermore, in addition to these specific cavity and crown sites, the individual charge groups of $\mathbf{1}$ and $\mathbf{2}$ can function as weak (pseudo-specific) binding sites for ions of opposite charge.

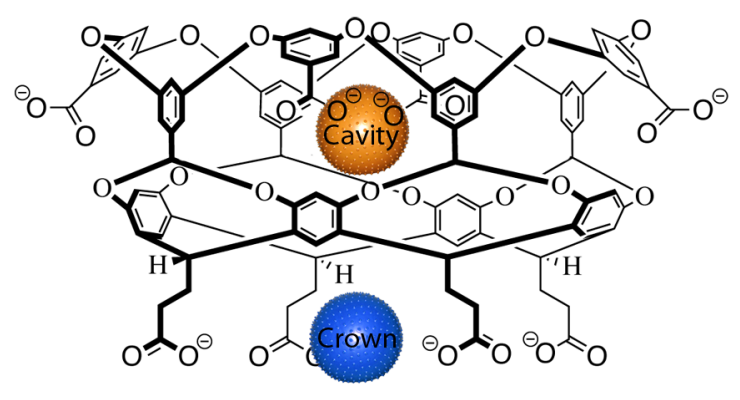

1

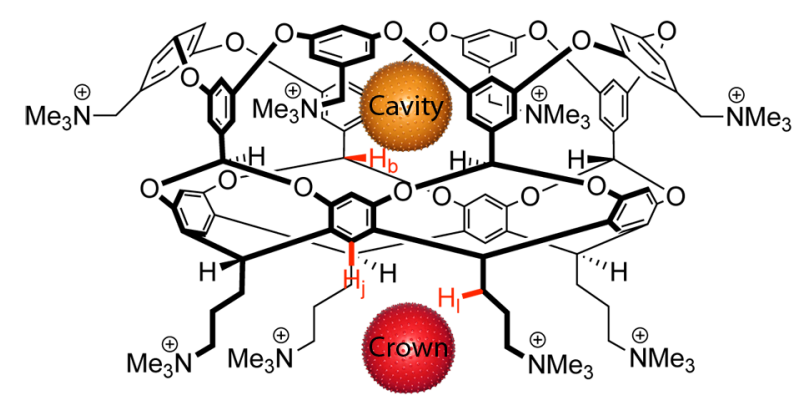

2

Figure 1: Chemical structures of octaacid 1 and positand 2 showing the anionic binding sites of the two hosts (orange and red) and the potential cationic binding site of 1 (blue). 


\section{Results and Discussion}

To determine the effects of salts on $\mathbf{1}$ (counter ion $\mathrm{Na}^{+}$) DLS was used to monitor its observed hydrodynamic volume during titration with various halide salts. The fifteen salts studied were a matrix of the alkali metal cations $\mathrm{Li}^{+}, \mathrm{Na}^{+}, \mathrm{K}^{+}$, and $\mathrm{Cs}^{+}$in combination with the halides $\mathrm{F}^{-}$through $\mathrm{I}^{-}$, the one omission being poorly soluble lithium fluoride (maximum solubility $=$ $0.134 \mathrm{~g} \mathrm{~mL}^{-1}$ ). Unsurprisingly, given the $\mathrm{p} K_{\mathrm{a}}$ values of carboxylic acids, host $\mathbf{1}$ has limited solubility in unbuffered water. Thus for solubility reasons, titrations of 1 were performed in $20 \mathrm{mM} \mathrm{NaOH}$ solution (see Supporting Information File 1, Figure S2, for more details). In each case titrations were taken to $100 \mathrm{mM}$ salt where it was assumed that the host is fully screened [21-23]. Figure 2 shows the effects of the different salts on the observed size of $\mathbf{1}$.

The reported hydrodynamic diameters were calculated using the Stokes-Einstein equation (Equation 1), which assumes host $\mathbf{1}$ is a spherical particle,

$$
D=\frac{k_{\mathrm{b}} T}{6 \pi \eta r_{\mathrm{H}}},
$$

where $D$ is the diffusion constant, $k_{\mathrm{b}}$ is the Boltzmann constant, $T$ is the temperature, $\eta$ is the viscosity of the solution, and $r_{\mathrm{H}}$ is the hydrodynamic radius.

In all cases, at the initial $20 \mathrm{mM}$ concentration of $\mathrm{NaOH}$ the light scattering induced by $\mathbf{1}$ was weak. This resulted in relatively flat autocorrelation functions generated from the measured fluctuations in scattered light. Consequently, the recorded size of the host was both anomalously small and highly variable, covering the range 1.0 to $1.7 \mathrm{~nm}$ (Figure 2). This compares to molecular models which show host $\mathbf{1}$ approximates to an anti-cube (square antiprism) with sides of $\approx 2.0 \mathrm{~nm}$. The weak light scattering of $\mathbf{1}$ was attributed to the high charge density of the host and the low ionic strength of the solution engendering significant Coulombic interactions between host molecules [24]. Titrating samples with the different salts led to much stronger light scattering and an apparent increase in the hydrodynamic diameter of the host to a more realistic $\approx 2 \mathrm{~nm}$. In all cases, however, the nature of the cation had no perceivable effect; each metal ion resulted in a hydrodynamic diameter for 1 of $2.1 \pm 0.2 \mathrm{~nm}$ (Table 1). The invariance in these results reveals the power of the carboxylate as a water-solubilizing

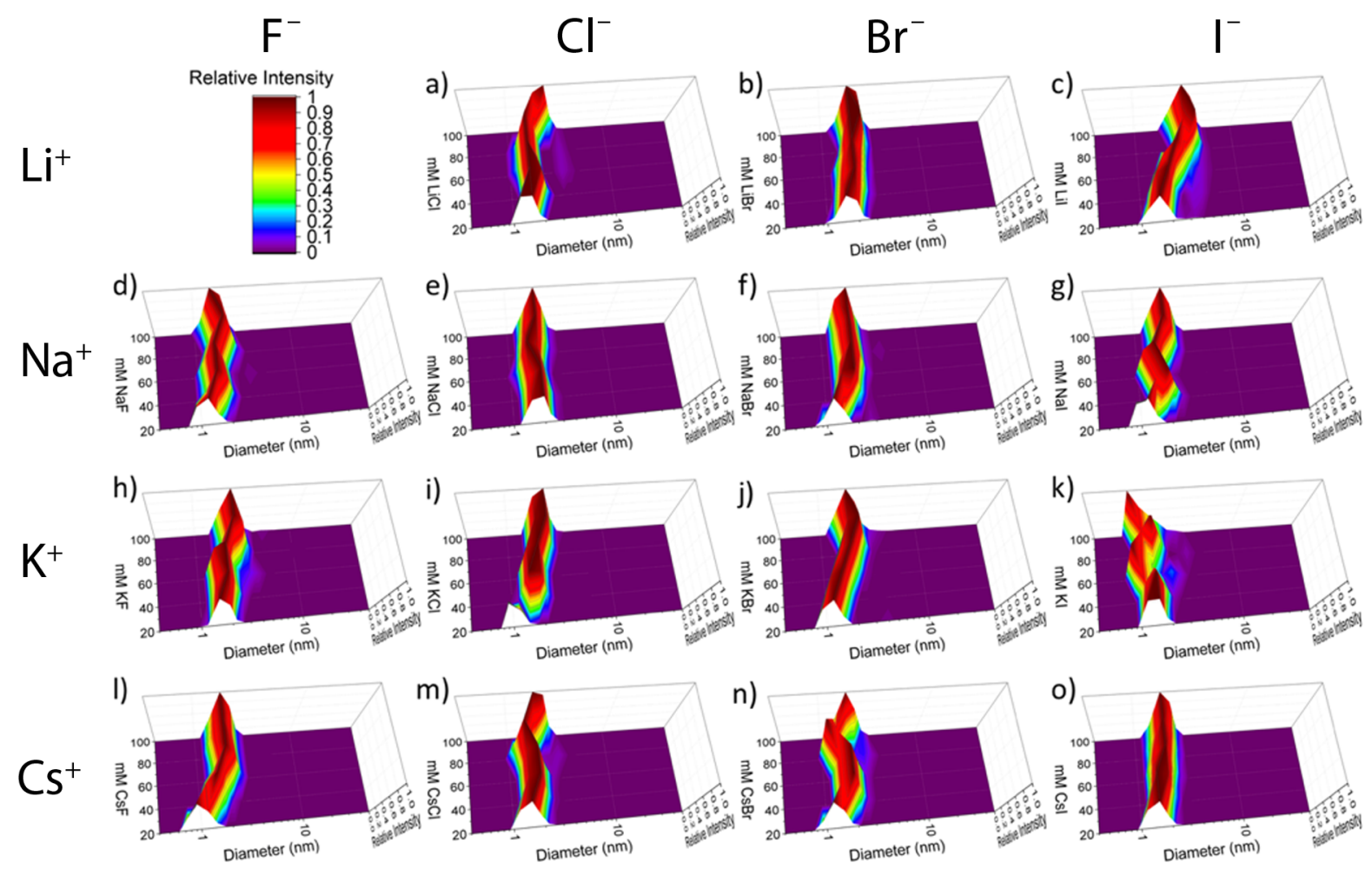

Figure 2: Representative plots of the volume-weighted distribution obtained by DLS for salts titrated into 2.00 mM 1: a) LiCl, b) LiBr, c) Lil, d) $\mathrm{NaF}$, e) $\mathrm{NaCl}$, f) $\mathrm{NaBr}$, g) $\mathrm{Nal}$, h) $\mathrm{KF}$, i) $\mathrm{KCl}$, j) $\mathrm{KBr}$, k) KI, l) CsF, m) CsCl, n) CsBr, o) Csl. Scale shown in the upper-left corner. The $x$-axis represents hydrodynamic diameter, the $y$-axis the concentration of the respective salt $(\mathrm{mM})$, and the $z$-axis the relative intensity. 


\begin{tabular}{|c|c|c|c|c|}
\hline cation & anion & host $1^{a, b} \max$ dia. $(n m)$ & host $2^{\mathrm{a}, \mathrm{b}}$ max. dia. $(\mathrm{nm})$ & n-mer aggregate ${ }^{b} \#$ (for host 2) \\
\hline \multirow{4}{*}{$\mathrm{Li}^{+}$} & $\mathrm{F}^{-}$ & $-^{c}$ & $-^{c}$ & \\
\hline & $\mathrm{Cl}^{-}$ & $2.1 \pm 0.0$ & $2.1 \pm 0.1$ & \\
\hline & $\mathrm{Br}^{-}$ & $2.1 \pm 0.2$ & $2.5 \pm 0.0$ & $1.7 \pm 0.0$ \\
\hline & $\mathrm{I}^{-}$ & $2.0 \pm 0.0$ & $14.3 \pm 0.5^{d}$ & $314 \pm 32^{d}$ \\
\hline \multirow{4}{*}{$\mathrm{Na}^{+}$} & $\mathrm{F}^{-}$ & $1.8 \pm 0.1$ & $1.8 \pm 0.3$ & \\
\hline & $\mathrm{Cl}^{-}$ & $2.0 \pm 0.2$ & $2.1 \pm 0.1$ & \\
\hline & $\mathrm{Br}^{-}$ & $1.8 \pm 0.0$ & $2.6 \pm 0.1$ & $1.8 \pm 0.1$ \\
\hline & $\mathrm{I}^{-}$ & $2.0 \pm 0.1$ & $18.6 \pm 3.1^{d}$ & $764 \pm 260^{d}$ \\
\hline \multirow{4}{*}{$\mathrm{K}^{+}$} & $\mathrm{F}^{-}$ & $2.1 \pm 0.4$ & $2.0 \pm 0.1$ & \\
\hline & $\mathrm{Cl}^{-}$ & $2.1 \pm 0.1$ & $2.0 \pm 0.0$ & \\
\hline & $\mathrm{Br}^{-}$ & $2.1 \pm 0.1$ & $2.4 \pm 0.1$ & $1.4 \pm 0.1$ \\
\hline & $\mathrm{I}^{-}$ & $2.2 \pm 0.3$ & $11.6 \pm 0.8$ & $170 \pm 37$ \\
\hline \multirow{4}{*}{$\mathrm{Cs}^{+}$} & $\mathrm{F}^{-}$ & $1.9 \pm 0.0$ & $2.0 \pm 0.3$ & \\
\hline & $\mathrm{Cl}^{-}$ & $2.0 \pm 0.1$ & $2.0 \pm 0.0$ & \\
\hline & $\mathrm{Br}^{-}$ & $1.9 \pm 0.1$ & $2.4 \pm 0.0$ & $1.5 \pm 0.0$ \\
\hline & $\mathrm{I}^{-}$ & $1.9 \pm 0.1$ & $11.9 \pm 0.2$ & $180 \pm 10$ \\
\hline
\end{tabular}

aDetermination of the maximum hydrodynamic diameter (max. dia.) was made regardless of the salt concentration at which the maximum size occurred. In the event of a bimodal distribution, the mode that accounted for $>10 \%$ of the total distribution and had the largest diameter was used to determine max. dia. ${ }^{b}$ Values are the average of two datasets. ${ }^{c}$ For solubility reasons titrations with LiF were not performed. ${ }^{d}$ Values are the average of three or more datasets.

group. Although its $\mathrm{p} K_{\mathrm{a}}$ may not be optimal for deprotonation at neutral or physiological $\mathrm{pH}$, its small size and relatively high free energy of hydration $\left(-373 \mathrm{~kJ} \mathrm{~mol}^{-1}\right)$ ensure that ion-pairing effects are not strong. This was further confirmed by ${ }^{1} \mathrm{H}$ NMR spectroscopy (Supporting Information File 1, Figure S3), which revealed only trace amounts of host aggregation $(\approx 5 \%)$. Furthermore, even at $100 \mathrm{mM}$ salt concentration, ${ }^{1} \mathrm{H}$ NMR spectroscopy failed to show any significant association of $\mathrm{Cs}^{+}$ to the crown of four carboxylates. Thus, although this crown is the most obvious potential cation binding site, we see no evidence of specific complexation here. More generally, despite the high charge density of $\mathbf{1}$, monovalent alkali metal ions cannot associate with it sufficiently to induce significant aggregation and a reverse Hofmeister effect. This was not, however, the case with divalent metal ions, which are well recognized to interact strongly with carboxylates and induce aggregation [25]. Thus, visual inspection upon the addition of $\mathrm{ZnCl}_{2}$ to give a $100 \mathrm{mM}$ salt concentration revealed extensive precipitation of the host. Returning to the point that the majority of anionic groups in biomacromolecules are strongly solvated, it is interesting to contemplate the idea that the prevalence of alkali metal ions in the environment exerted evolutionary pressures on biomacromolecules to select carboxylate, phosphates and sulfates and hence minimize ion pairing, charge neutralization, and deleterious precipitation effects in living systems.
Overall, octacarboxylate $\mathbf{1}$ is a binder of large, polarizable anions in its non-polar pocket [12], but is not a perceptible binder of alkali metal cations. Building on this, we carried out similar DLS studies with host 2 (counter ion $\mathrm{Cl}^{-}$) using the same aforementioned salts (Figure 3). In a previous work, our DLS studies of this host involved solutions buffered with $40 \mathrm{mM}$ phosphate ( $\mathrm{pH}$ 7.3) [14]. Under these conditions, the initially measured sizes in the absence of added salt were consistently $1.9-2.1 \mathrm{~nm}$; values that match the modeling of the host. In stark contrast to this earlier work, but analogously to host $\mathbf{1}$, when we examined solutions of $\mathbf{2}$ in the absence of any added buffer and salt, light scattering was weak. This resulted in flat autocorrelation functions and again an anomalously small and highly variable hydrodynamic volume $(0.5-1.4 \mathrm{~nm})$. This issue noted, at the titration point of $80 \mathrm{mM}$ salt the curvature of the autocorrelation function greatly increased, and the observed hydrodynamic diameter approached the expected $\approx 2.0 \mathrm{~nm}$. Hence although for all of the studies here the starting point for each titration was $20 \mathrm{mM}$ salt, the first data point for each titration was ignored.

An obvious trend in the data for host 2 (Figure 3) is how the nature of the halide affects aggregation. Over all concentrations of $\mathrm{F}^{-}$salts the hydrodynamic diameter of the host was anomalously small, with maximum diameters measured in the pres- 


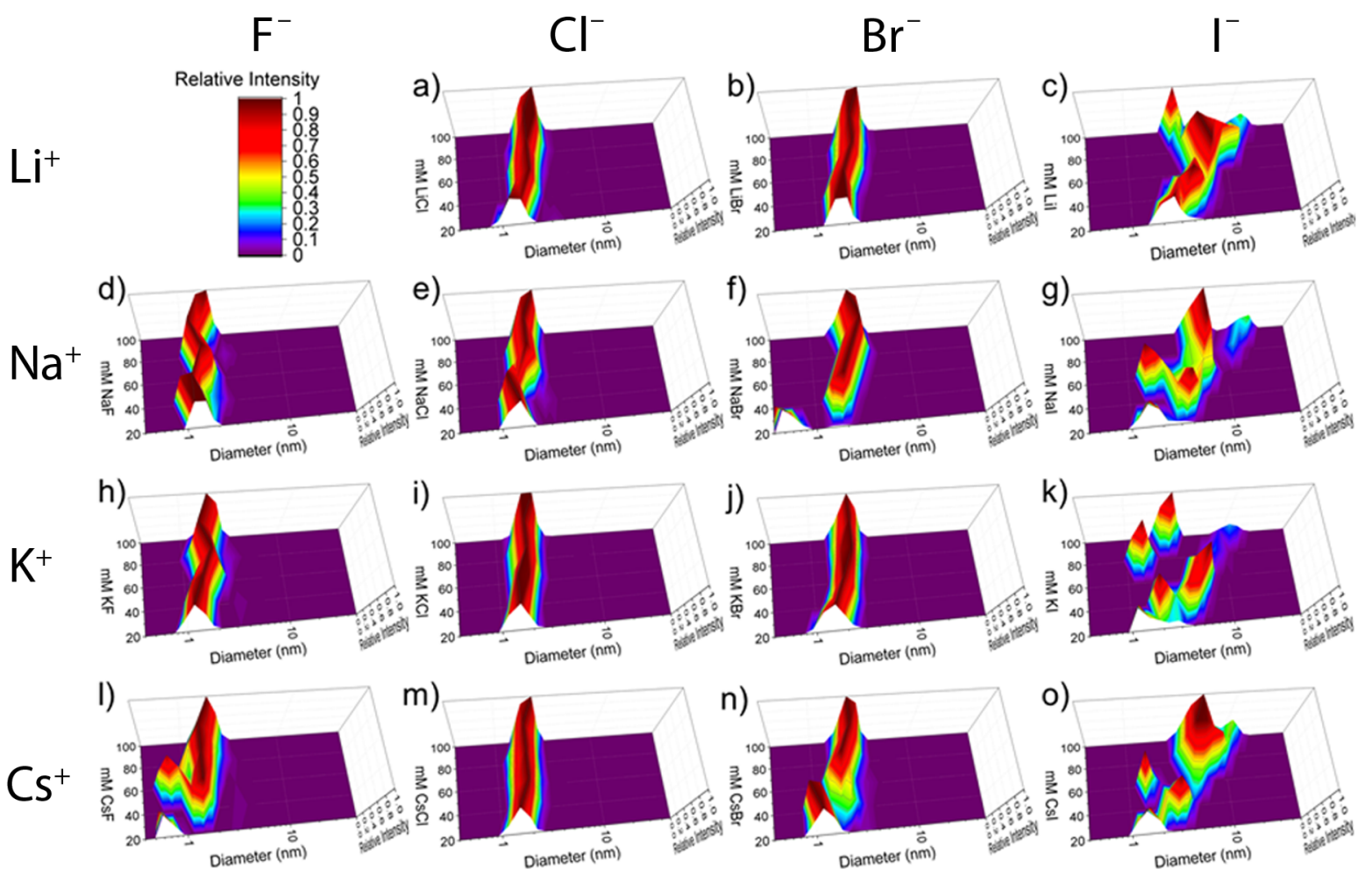

Figure 3: Representative plots of the volume-weighted distribution obtained by DLS for salts titrated into 2.00 $\mathrm{mM} \mathrm{2:} \mathrm{a)} \mathrm{LiCl,} \mathrm{b)} \mathrm{LiBr}$, c) Lil, d) $\mathrm{NaF}$, e) $\mathrm{NaCl}$, f) $\mathrm{NaBr}$, g) $\mathrm{Nal}$, h) $\mathrm{KF}$, i) $\mathrm{KCl}$, j) $\mathrm{KBr}$, k) KI, I) CsF, m) CsCl, n) $\mathrm{CsBr}$, o) Csl. Scale shown in the upper left corner. The $x$-axis represents hydrodynamic diameter, the $y$-axis the concentration of the respective salt $(\mathrm{mM})$, and the $z$-axis the relative intensity.

ence of $\mathrm{NaF}, \mathrm{KF}$, and $\mathrm{CsF}$ being 1.6, 1.9, and $1.8 \mathrm{~nm}$. The strongly solvated $\mathrm{F}^{-}$ion [26] has a very weak affinity for host $\mathbf{2}$ [14], and we therefore interpret these small hydrodynamic diameters to limited binding to host $\mathbf{2}$ and hence an inability to screen interhost interactions. In contrast, in the presence of at least $80 \mathrm{mM} \mathrm{Cl}^{-}$salts the hydrodynamic diameter of host $\mathbf{2}$ was consistently within the expected range of $2.0-2.1 \mathrm{~nm}$. Thus, independent of the metal cation $\mathrm{Cl}^{-}$is an ideal anion for effectively screening intermolecular charge-charge interactions between the host (Table 1). The case of $\mathrm{Br}^{-}$was quite different. For all salts, the addition of $\mathrm{Br}^{-}$leads to an increase in the hydrodynamic diameter, with $\mathrm{NaBr}$ giving the largest increase to $2.6 \mathrm{~nm}$. This corresponds to the formation of a dimer aggregate. Aggregation was even more extreme with the $\mathrm{I}^{-}$salts. All $\mathrm{I}^{-}$salts caused extensive aggregation of the host, and a determination of the maximum size induced by the four salts ranged from 170 and 180-mers in the presence of KI and CsI, to $\approx 724$-mers for $\mathrm{NaI}$. Evidently the difference in the free energies of solvation of $\mathrm{Br}^{-}$and $\mathrm{I}^{-}\left(\Delta G_{\text {hyd }}=-321\right.$ and $-283 \mathrm{~kJ} \mathrm{~mol}^{-1}$, respectively) is key to allowing more ion pairing between the trimethylammonium groups of $\mathbf{2}$ and $\mathrm{I}^{-}$to induce substantial aggregation.
The data for the $\mathrm{I}^{-}$salts illustrate a further complexity to the ability of salts to induce precipitation of ammonium ions such as $\mathbf{2}$. Thus, although the aggregation induced by KI and CsI are not significantly different, there is a trend for cation-induced aggregation of cationic 2 : namely $\mathrm{Na}^{+}>\mathrm{Li}^{+}>\mathrm{K}^{+} \approx \mathrm{Cs}^{+}$. This cation effect must be indirect. If the counter ions of $\mathbf{2}\left(\mathrm{Cl}^{-}\right)$are viewed as non-coordinating, this phenomenon can be interpreted as arising from a simple competition between the two "hosts" 2 and $\mathrm{M}^{+}$(Scheme 1). In such a system, $\mathrm{I}^{-}$can only associate with host $\mathbf{2}$ and induce charge neutralization and aggregation when it is in the free state, but if it itself strongly associates with the counter ion of the salt then it will not be able to bind strongly to 2 .

$$
2 \cdot \frac{+2}{-2} \quad I^{-} \frac{+M^{+}}{-M^{+}} \quad M I
$$

Scheme 1: Visualization of the competitive equilibrium between iodide binding to host 2 or associating with its alkali metal cation $\mathrm{M}^{+}$. Non-competing $\mathrm{Cl}^{-}$is omitted for simplicity. 
There is a well-established "volcano plot" relationship between the standard heat of solution of crystalline alkali halides and the difference between the absolute free energy (or heat) of hydration of the corresponding anion and cation $[27,28]$. As a result, in the words of Fajans, "in the case of alkali halides, the solubility in a number of salts with the same cation (anion) and different anions (cations) is at a minimum when the cation and anion are approximately equal and increases with increasing difference of the ionic radii" [29]. This has been built upon by Collins who proposed that the difference in heats of hydration of a cation and anion is a surrogate for the extent of anion pairing in solution; that small (large) anions preferentially bind with small (large) cations, whereas large size differences lead to weak association [30]. Thus, this law of matching water affinities (LMWA) suggests that in aqueous solution CsI is more strongly ion-paired than LiI. The observed trend in the aggregation of $2(\mathrm{NaI}>\mathrm{LiI}>\mathrm{KI} \approx \mathrm{CsI})$ is therefore not in full agreement with the LMWA. The LMWA correlates with our data that $\mathrm{K}^{+}$and $\mathrm{Cs}^{+}$should pair strongly with $\mathrm{I}^{-}$and therefore induce weak aggregation. However, it also predicts that $\mathrm{Li}^{+}$and $\mathrm{I}^{-}$ should form the weakest ion pair and that therefore LiI should be the greatest aggregator of $\mathbf{2}$. As Table 1 reveals, this is not the case; it is $\mathrm{NaI}$ that has the strongest influence on the host.

A straightforward answer for this may be that entropy is a part of the aggregation of $\mathbf{2}$, whereas the LMWA is purely enthalpically based. Additionally, however, the absolute heats of hydration of anions and cations calculated by Morris make many assumptions, and in part rely on models that assume ideality for their determinations.

Cation effects for the bromide salts are less pronounced than those of the iodide salts, nevertheless there are small but significant differences between the pairs of cations $\mathrm{Li}^{+} / \mathrm{Na}^{+}$and $\mathrm{K}^{+} / \mathrm{Cs}^{+}$. The former pair, as would be expected considering the data for iodide salts, leads to greater aggregation than that observed with the potassium and cesium salts. These results reveal that in contrast to host $\mathbf{1}$, the weakly solvated groups of $\mathbf{2}$ result in significant ion-pairing effects that can, in extreme cases such as $\mathrm{I}^{-}$salts, lead to a pronounced reverse Hofmeister effect. Importantly, our DLS studies reveal that this effect is also influenced indirectly by the nature of the cation of the salt.

\section{Conclusion}

Dynamic light scattering reveals the ion-specific interactions of carboxylate and trimethylammonium groups, and hence the inherent asymmetry between negatively and positively charged molecules. The negatively charged solubilizing groups of host $\mathbf{1}$ are relatively strongly solvated, so much so that the nature of the alkali metal cation has very little effect on aggregation. Divalent metal ions are required to induce aggregation in this host. In contrast, the more weakly solvated charged groups of $\mathbf{2}$ allow ion-specific interactions with halide anions. Specifically, weakly solvated $\mathrm{I}^{-}$, and to a lesser extent $\mathrm{Br}^{-}$, can associate closely with host $\mathbf{2}$, induce charge neutralization, and hence bring about aggregation. Importantly, because of the power of $\mathrm{I}^{-}$to induce aggregation in $\mathbf{2}$ it is even possible to observe how ion pairing within a salt can influence its aggregation ability. Considering the ubiquity of alkali metal halide ions in Nature, we are examining other systems to provide greater detailing of how the balance of ion pairing in two-cation/two-anion systems influences Hofmeister effects.

\section{Experimental}

Reagents were purchased from the commercial supplier SigmaAldrich Corp. and were used without further purification. Deuterated solvents were purchased from Cambridge Isotopes and used without further purification. Hosts $\mathbf{1}$ and $\mathbf{2}$ were synthesized by the procedures reported previously [15,16,31]. All ${ }^{1} \mathrm{H}$ NMR spectra were collected on a Bruker $500 \mathrm{MHz}$ spectrometer at $25{ }^{\circ} \mathrm{C}$. Spectral processing was performed using Mnova software (Mestrelab Research, S.L.). All dynamic light scattering measurements were performed on a Nicomp ZLS Z3000 particle size analyzer (Particle Sizing Systems - Port Richey, FL), with a $50 \mathrm{~mW}$ laser diode (660 nm wavelength) and an avalanche photodiode (APD) detector. Measurements of scattered light were made at $90^{\circ}$, with data collected at $23{ }^{\circ} \mathrm{C}$ and processed using a non-negative least squares Nicomp analysis.

\section{DLS solution preparation and analysis procedures}

All solutions of 1 were prepared in $20.0 \mathrm{mM} \mathrm{NaOH}$ in 18.2 $\mathrm{M} \Omega \cdot \mathrm{cm}$ Milli-Q $\mathrm{H}_{2} \mathrm{O}$. Solutions of 2 were prepared in unbuffered 18.2 M $\Omega \cdot \mathrm{cm}$ Milli-Q $\mathrm{H}_{2} \mathrm{O}$. All host solutions were prepared at a concentration of $2.00 \mathrm{mM}$. Solutions of $\mathbf{1}$ and $\mathbf{2}$ were titrated with a $2.00 \mathrm{M}$ salt solution in aliquots of $20 \mathrm{mM}$ until reaching a final concentration of $100 \mathrm{mM}$ (50 equiv) salt. Dilution of the host solution during the titration was maintained at $<5 \%$ for all titrations.

Samples were centrifuged for $10 \mathrm{~min}$ at $10,000 \mathrm{rpm}$ prior to each titration but not centrifuged thereafter. Solutions of host were titrated with salt, then shaken and vortexed to ensure mixing before acquiring DLS measurements. For each data point in a titration, analyses were performed in quadruplicate at a channel width of $5 \mu \mathrm{s}$. Particularly, at low salt concentrations, weak light scattering resulted in a flat autocorrelation curve; this data was immediately discarded. Of the remaining data, that with the lowest fit error was kept. At every salt concentration, the data was replicated a minimum of one time using a separate solution of host. Those data were then averaged and presented 
herein. Results shown are representative of the volumeweighted distribution. Surface plots of the raw, volumeweighted distribution data were plotted using OriginPro software.

\section{NMR solution sample preparation and analysis procedures}

Monodispersity of the host $\mathbf{1}$ solution was confirmed by Pulsed Gradient Spin Echo (PGSE) NMR (Supporting Information File 1, Figure $\mathrm{S} 1)$ in $\mathrm{H}_{2} \mathrm{O}$ locked with $\mathrm{D}_{2} \mathrm{O}$ in a $5 \mathrm{~mm}$ coaxial capillary insert (Wilmad-Labglass - Vineland, NJ). The concentration of the stock solid was determined by titration in triplicate with a $25.0 \mathrm{mM}$ sodium ethanesulfonate (SES) solution, and integration of the methyl or methylene peaks of ethanesulfonate and the $\mathrm{H}_{1}$ peak of the host.

Solutions for NMR titrations were prepared in $13.0 \mathrm{mM} \mathrm{NaOH}$ in $\mathrm{D}_{2} \mathrm{O}$ (Supporting Information File 1, Figure S2). Titrations of the host were carried out with $2.0 \mathrm{mM}$ host solutions. Stock solutions of $\mathrm{NaOH}$ were prepared at $286.0 \mathrm{mM}$. An aliquot of $0.5 \mathrm{~mL}$ of host was titrated in an NMR tube with careful addition of small aliquots of $\mathrm{NaOH}$. Analysis of $\mathbf{1}$ with $\mathrm{CsCl}$ was performed by the addition of a $2.00 \mathrm{M} \mathrm{CsCl}$ solution to a $2.0 \mathrm{mM}$ host solution such that the final $\mathrm{CsCl}$ concentration was $100 \mathrm{mM}$ (50 equiv) and dilution of the host was 5\% (Supporting Information File 1, Figure S3).

\section{Supporting Information}

\section{Supporting Information File 1}

Additional analytical data and NMR spectra.

[https://www.beilstein-journals.org/bjoc/content/ supplementary/1860-5397-14-195-S1.pdf]

\section{Acknowledgements}

The authors gratefully acknowledge the support of the National Institutes of Health (GM 098141). A.W. also acknowledges the Louisiana Board of Regents for a graduate student fellowship (LEQSF(2013-18)-GF-13).

\section{ORCID ${ }^{\circledR}$ iDs}

Anthony Wishard - https://orcid.org/0000-0003-2265-2053 Bruce C. Gibb - https://orcid.org/0000-0002-4478-4084

\section{References}

1. Chan, C. W.; Smith, D. K. Supramol. Chem. 2017, 29, 688-695. doi:10.1080/10610278.2016.1234711

2. Debye, P.; Hückel, E. Phys. Z. 1923, 24, 185-206.

3. Honig, B.; Nicholls, A. Science 1995, 268, 1144-1149. doi:10.1126/science. 7761829
4. Salis, A.; Ninham, B. W. Chem. Soc. Rev. 2014, 43, 7358-7377. doi:10.1039/c4cs00144c

5. Buck, R. P.; Rondinini, S.; Covington, A. K.; Baucke, F. G. K.; Brett, C. M. A.; Camoes, M. F.; Milton, M. J. T.; Mussini, T.; Naumann, R.; Pratt, K. W.; Spitzer, P.; Wilson, G. S. Pure Appl. Chem. 2002, 74, 2169-2200. doi:10.1351/pac200274112169

6. Liang, Y.; Hilal, N.; Langston, P.; Starov, V. Adv. Colloid Interface Sci. 2007, 134-135, 151-166. doi:10.1016/j.cis.2007.04.003

7. Bostrom, M.; Lima, E. R. A.; Tavares, F. W.; Ninham, B. W. J. Chem. Phys. 2008, 128, 135104. doi:10.1063/1.2873307

8. Hofmeister, F. Arch. Exp. Pathol. Pharmakol. 1888, 24, 247-260. doi:10.1007/bf01918191

9. Kunz, W.; Henle, J.; Ninham, B. W. Curr. Opin. Colloid Interface Sci. 2004, 9, 19-37. doi:10.1016/j.cocis.2004.05.005

10. Jungwirth, P.; Cremer, P. S. Nat. Chem. 2014, 6, 261-263. doi:10.1038/nchem.1899

11. Collins, K. D.; Washabaugh, M. W. Q. Rev. Biophys. 1985, 18, 323-422. doi:10.1017/s0033583500005369

12. Carnegie, R. S.; Gibb, C. L. D.; Gibb, B. C. Angew. Chem., Int. Ed. 2014, 53, 11498-11500. doi:10.1002/anie.201405796

13. Sokkalingam, P.; Shraberg, J.; Rick, S. W.; Gibb, B. C. J. Am. Chem. Soc. 2016, 138, 48-51. doi:10.1021/jacs.5b10937 14. Jordan, J. H.; Gibb, C. L. D.; Wishard, A.; Pham, T.; Gibb, B. C. J. Am. Chem. Soc. 2018, 140, 4092-4099. doi:10.1021/jacs.8b00196

15. Gibb, C. L. D.; Gibb, B. C. J. Am. Chem. Soc. 2004, 126, 11408-11409. doi:10.1021/ja0475611

16. Liu, S.; Whisenhunt-loup, S. E.; Gibb, C. L. D.; Gibb, B. C. Supramol. Chem. 2011, 23, 480-485. doi:10.1080/10610278.2010.550290

17. Roland, C. D.; Li, H.; Abboud, K. A.; Wagener, K. B.; Veige, A. S. Nat. Chem. 2016, 8, 791-796. doi:10.1038/nchem.2516

18. Deshmukh, S. A.; Solomon, L. A.; Kamath, G.; Fry, H. C.; Sankaranarayanan, S. K. R. S. Nat. Commun. 2016, 7, No. 12367. doi:10.1038/ncomms 12367

19. Sato, K.; Ji, W.; Palmer, L. C.; Weber, B.; Barz, M.; Stupp, S. I. J. Am. Chem. Soc. 2017, 139, 8995-9000. doi:10.1021/jacs.7b03878

20. Chen, S.; Polen, S. M.; Wang, L.; Yamasaki, M.; Hadad, C. M.; Badjić, J. D. J. Am. Chem. Soc. 2016, 138, 11312-11317. doi:10.1021/jacs.6b06562

21. Boström, M.; Tavares, F. W.; Bratko, D.; Ninham, B. W. Ion Specific Interactions Between Pairs of Nanometer Sized Particles in Aqueous Solutions. In Smart Colloidal Materials. Progress in Colloid and Polymer Science; Richtering, W., Ed.; Springer: Berlin, 2006; Vol. 133, pp 74-77. doi:10.1007/2882_060

22. Salis, A.; Pinna, M. C.; Bilaničová, D.; Monduzzi, M.; Lo Nostro, P.; Ninham, B. W. J. Phys. Chem. B 2006, 110, 2949-2956. doi:10.1021/jp0546296

23. Smith, A. M.; Lee, A. A.; Perkin, S. J. Phys. Chem. Lett. 2016, 7, 2157-2163. doi:10.1021/acs.jpclett.6b00867

24. Hassan, P. A.; Rana, S.; Verma, G. Langmuir 2015, 31, 3-12. doi:10.1021/la501789z

25. Kherb, J.; Flores, S. C.; Cremer, P. S. J. Phys. Chem. B 2012, 116, 7389-7397. doi:10.1021/jp212243c

26. Marcus, Y. Ion Properties; Marcel Dekker, Inc.: New York, 1997; p 259.

27. Morris, D. F. C. Struct. Bonding 1968, 4, 63-82. doi:10.1007/BFb0119185

28. Morris, D. F. C. Struct. Bonding 1969, 6, 157-159. doi:10.1007/BFb0118857

29. Fajans, K. Naturwissenschaften 1921, 9, 729-738. doi:10.1007/bf01487182 
30. Collins, K. D. Biophys. J. 1997, 72, 65-76.

doi:10.1016/s0006-3495(97)78647-8

31. Hillyer, M. B.; Gibb, C. L. D.; Sokkalingam, P.; Jordan, J. H.; loup, S. E.; Gibb, B. C. Org. Lett. 2016, 18, 4048-4051.

doi:10.1021/acs.orglett.6b01903

\section{License and Terms}

This is an Open Access article under the terms of the Creative Commons Attribution License

(http://creativecommons.org/licenses/by/4.0). Please note that the reuse, redistribution and reproduction in particular requires that the authors and source are credited.

The license is subject to the Beilstein Journal of Organic Chemistry terms and conditions:

(https://www.beilstein-journals.org/bjoc)

The definitive version of this article is the electronic one which can be found at: doi:10.3762/bjoc. 14.195 